\title{
Higher Cognitive Abilities (Toward a Physical Theory of the Mind)
}

\author{
Leonid I. Perlovsky, Senior Member, IEEE
}

\begin{abstract}
Higher cognitive abilities of the mind are described mathematically. Few "first principles" are elucidated, including the knowledge instinct, an inborn drive to increase the knowledge. It leads to understanding of higher cognitive abilities of the mind, including conceptual thinking, emotional evaluation, consciousness and unconscious, intuition, imagination, experiences of beautiful and sublime. The mathematical descriptions correspond to intuition, psychological and neural data, philosophical analyses. We discuss theoretical predictions and testing of the theory.
\end{abstract}

\section{ABILITIES OF THE MIND}

$\mathrm{P}$ lato asked how is it possible that unlearned people could understand complexity of the world $\left[{ }^{1}\right]$. He came to a conclusion that the human mind is connected to the a priori world of ideas $(\varepsilon 1 \delta \varepsilon)$, and these ideas are the foundation for our understanding. Today, we know that the mind understands the world by relying on internal representations, or models of the world that were learned previously, an hour ago, earlier in life, in childhood, and ultimately, relying on the inborn genetic information. These internal models of the mind are related to Plato's ideas $\left[{ }^{2}\right]$, Aristotelian forms $\left[{ }^{3}, 4\right]$, Kantian ability for understanding $\left[{ }^{5},{ }^{2}\right]$, Jungian archetypes $\left[{ }^{6}, 2\right]$, and various mechanisms of concepts discussed in artificial intelligence, psychology, cognitive science and neural networks $\left[{ }^{7},{ }^{8}\right]$. The mind brings concept-models in correspondence to objects and situations in the world; in the result, there appear phenomena, internal mind's perceptions (or representations, which could be conscious or unconscious to varying degrees).

The definitions of instincts, concepts, emotions, are the subject of research and debate, while theories of life and intelligence are in the development. Here I introduce these notions briefly for the purpose of connecting discussions in psychology, philosophy and neurobiology to a mathematical description. Further discussions and references can be found in $\left.{ }^{9}\right]$. Concepts (say, a word "chair", written or spoken) are very different from objects (a chair one sits on). In our brain there are inborn structures that were developed over hundreds of millions of years of evolution specifically to enable fast learning by combining into a single conceptmodel a spoken, imagined and real chair. Let's note that the "real chair" is what is seen by our eyes, but also what is sensed as a "seat" by the sitting part of our body. Therefore "chair" is a bodily-sensed-spatio-thought concept. The process of comparing concept-models to objects around us is not simple, nor straightforward. An ability for finding

L. Perlovsky is with the Air Force Research Laboratory, SNHE, 80 Scott Drive, Hanscom AFB, MA 01731, phone: 781-377-1728, fax: 781-3778984, e-mail: leonid.perlovsky@ hanscom.af.mil. correspondence between concepts and objects Kant called judgment $\left[{ }^{10}\right]$; he identified this ability as a foundation for all higher spiritual abilities of our mind.

An ability for concept-models evolved during evolution to enhance survivability; it works together with other mechanisms developed for this purpose, first of all, instincts and emotions. Instincts are like internal sensors that generate signals in neural networks indicating the basic needs of an organism, say hunger $\left[{ }^{11}\right]$. Connection of instincts and concepts is accomplished by emotions. In a usual conversation, «emotions» refer to a special type of behavior: agitation, higher voice pitch, bright eyes, but these are just shows of emotions. Emotions are evaluations «goodbad». Evaluations not according to concepts of good and bad, but direct instinctive evaluations better characterized in terms of pleasure or pain. An emotion evaluates a degree to which a phenomenon (objects or a situation) satisfies our instinctual needs. Emotions are signals in neural pathways that carry information about object values from instinctrelated brain areas to perceptual, cognitive, decision-making and behavior-generating areas. Emotions «mark» perceived phenomena with their values for instinct satisfaction. There are inborn as well as learned emotional responses. A mathematical description of «marking» of concepts by emotions was first obtained by Grossberg and co-workers in the late 1980 s, e.g., $\left[{ }^{12}\right]$. A review of discussions of the nature of emotions can be found in $\left[{ }^{9}\right]$.

Every instinct generates evaluative emotional signals indicating satisfaction or dissatisfaction of this instinct. Therefore emotions are called evaluative signals. These signals affect the process of comparing concept-models to objects around us (this explains why a hungry person "sees food all around"). So, instinctual needs affect our perception and cognition through emotions; and model-concepts formed in evolution and culture originally are intended for survival and therefore for instinct satisfaction. This intentionality of the mind was a subject of much discussions and controversies $\left[{ }^{13},{ }^{14}, 9\right]$. Many emotions originate in ancient parts of the brain, relating us to primates and even to lower animals $\left[{ }^{15}\right]$. Ability for concepts includes learning and recognition (that is creating and remembering models as well as recognizing objects and situations more-or-less corresponding to earlier learned models). In perception and recognition processes concept-models should always be modified and adapted to the actual conditions around. These adaptation processes are driven by the knowledge instinct. This paper will be mostly concerned just with one type of behavior, the behavior of improving knowledge. 
The next Section 2 contains a mathematical formulation of the theory of neural modeling fields and the knowledge instinct. This gives a foundation for an initial description of the mind abilities discussed above and also touches on relationships to psychological and neurophysiological data. Section 3 discusses relationships between the theory and concepts of the mind originated in multiple disciplines as well as future directions of experimental programs and theoretical development towards the physical theory of the mind.

\section{THE KNOWLEDGE INSTINCT}

A mathematical theory in this section describes operations of the human mind driven by the instinct for knowledge. Exploratory behavior of humans and animals was noticed by biologists, even when basic bodily needs, like eating, were satisfied. Biologists and psychologists discussed curiosity in this regard (Berlyne, 1960; 1973). However, it was not mentioned among 'basic instincts' on a par with instincts for food and procreation. The reasons were that it was difficult to define, and that its fundamental nature was not obvious. The fundamental nature of this mechanism became clear when mathematical description of the mind were attempted. Virtually all learning and adaptive algorithms (tens of thousands of publications) maximize correspondence between the algorithm internal structure (knowledge in a wide sense) and objects of recognition. The reason is that our knowledge always has to be modified to fit the current situations. One rarely sees exactly the same object: illumination, angles, surrounding objects are usually different; therefore, adaptation-learning is required. A mathematical formulation of the mind mechanisms makes obvious the fundamental nature of our desire for knowledge. The mathematical formulation of the knowledge instinct is given in this section.

Another important principle of brain organization indicated by psychological and neuro-physiological data is a hierarchical organization $\left[{ }^{15}\right]$. This hierarchy is not strict: lateral connections among modules are abound; and this hierarchy is not one-directional: extensive feedback connections relate "higher" levels back to "lower" levels (e.g., an eye nerve connecting retina to visual cortex has more feedback connections, from the cortex to the retina, than feedforward ones, from the retina to the cortex). Existence of feedback connections was appreciated for a while, and this gave rise to an idea of combining "top-down" processing with the "bottom-up" one. Yet, the mathematical nature of the mechanisms of these connections was subject of many controversies $\left[{ }^{2}\right]$. This section describes a basic mechanism of interaction between two adjacent hierarchical levels of signals (fields of neural activation); sometimes, it will be more convenient to talk about these two signal-levels as an input to and output from a (single) processing-level. At each level, the output are concepts recognized (or formed) from input signals. At the bottom of the mind hierarchy, the input is sensory signals, the activation levels of sensory cells, and the output are "concepts" formed or recognized at this level; this output neuronal field of activated "concepts" serves as input to the next level; higher up the hierarchy, the inputs are objects or situations recognized at lower levels and outputs are more complex, more general situationconcepts or relationship-concepts.

A combination of the knowledge instinct and hierarchical organization is a foundation of our higher cognitive abilities.

\section{Internal Models, Learning, And Similarity}

First, we give a mathematical description of a single level in the mind hierarchy. Input signals to each level $\{\mathbf{X}(n)\}$, is a field of input neuronal synapse activations, $n$ enumerates the input neurons and $\mathbf{X}(\mathrm{n})$ are the activation levels; a set of concept-models $\{\mathrm{h}\}$ is characterized by the models (representations) $\left\{\mathbf{M}_{\mathrm{h}}(\mathrm{n})\right\}$ of the signals $\mathbf{X}(\mathrm{n})$; each model depends on its parameters $\left\{\mathbf{S}_{\mathrm{h}}\right\}$. In a highly simplified description of a visual cortex, $\mathrm{n}$ enumerates the visual cortex neurons, $\mathbf{X}(\mathrm{n})$ are the "bottom-up" activation levels of these neurons coming from the retina through visual nerve, and $\mathbf{M}_{h}(\mathrm{n})$ are the "top-down" activation levels (or priming) of the visual cortex neurons from previously learned objectmodels ${ }^{16}$. The learning process attempts to "match" these top-down and bottom-up activations by selecting "best" models and their parameters. This process is driven by the knowledge instinct, the inborn drive to improve correspondence between the models and the data. Mathematically, the knowledge instinct drives us to increase a similarity measure between the sets of models and signals, $\mathrm{L}\left(\{\mathbf{X}(\mathrm{n})\},\left\{\mathbf{M}_{\mathrm{h}}(\mathrm{n})\right\}\right)$. The similarity measure is a function of model parameters and associations between the input synapses and concepts-models. It is constructed in such a way that any of a large number of objects can be recognized, no matter if they appear on the left or on the right. Correspondingly, a similarity measure is designed so that it treats each concept-model as an alternative for each subset of signals

$$
\mathrm{L}(\{\mathbf{X}\},\{\mathbf{M}\})=\prod_{n \in N} \sum_{h \in H} \mathrm{r}(\mathrm{h}) 1\left(\mathbf{X}(\mathrm{n}) \mid \mathbf{M}_{\mathrm{h}}(\mathrm{n})\right) ;
$$

here $1\left(\mathbf{X}(\mathrm{n}) \mid \mathbf{M}_{\mathrm{h}}(\mathrm{n})\right)$ (or simply $1(\mathrm{n} \mid \mathrm{h})$ ) is a conditional partial similarity between one signal $\mathbf{X}(\mathrm{n})$ and one model $\mathbf{M}_{\mathrm{h}}(\mathrm{n})$, and all possible combinations of signals and models are accounted for in this expression. Parameters $r(h)$ are proportional to the number of signals $\{n\}$ associated with the model h.

In the process of learning, concept-models are constantly modified. From time to time a system forms a new concept, while retaining an old one as well; alternatively, old concepts are sometimes merged. Formation of new concepts and merging of old ones require a modification of the similarity measure (1); the reason is that more models always result in a better fit between the models and data. 
This is a well known problem, it can be addressed by reducing (1) using a "penalty function", $\mathrm{p}(\mathrm{N}, \mathrm{M})$ that grows with the number of models $\mathrm{M}$, and this growth is steeper for a smaller amount of data N. For example, an asymptotically unbiased maximum likelihood estimation leads to multiplicative $\mathrm{p}(\mathrm{N}, \mathrm{M})=\exp \left(-\mathrm{N}_{\mathrm{par}} / 2\right)$, where $\mathrm{N}_{\text {par }}$ is a total number of adaptive parameters in all models (this penalty function is known as Akaike Information Criterion, see $\left[^{2}\right]$ for further discussion and references).

\section{Fuzzy Dynamic Logic and MFT}

The learning process consists in estimating model parameters $\mathbf{S}_{\mathrm{h}}$ and associating subsets of signals with concepts by maximizing the similarity (1). Note, that (1) contains a large number of combinations of models and signals, a total of $\mathrm{H}^{\mathrm{N}}$ items; this was a cause for the combinatorial complexity of the past algorithms discussed in detail later. Modeling field theory (MFT) solves this problem by utilizing fuzzy dynamic logic $\left[{ }^{2,17}\right]$. MFT introduces association variables $\mathrm{f}(\mathrm{h} / \mathrm{n})$

$\mathrm{f}(\mathrm{h} \mid \mathrm{n})=\mathrm{r}(\mathrm{h}) \mathrm{l}(\mathrm{n} \mid \mathrm{h}) / \sum_{h^{\prime} \in H} \mathrm{r}\left(\mathrm{h}^{\prime}\right) 1\left(\mathrm{n} \mid \mathrm{h}^{\prime}\right)$.

These variables give a measure of correspondence between a signal $\mathbf{X}(\mathrm{n})$ and a model $\mathbf{M}_{\mathrm{h}}$ relative to all other models, $\mathrm{h}$.

A mechanism, an internal dynamics, of the Modeling Fields (MF) is defined as follows,

$$
\begin{aligned}
& \mathrm{df}(\mathrm{h} \mid \mathrm{n}) / \mathrm{dt}=\mathrm{f}(\mathrm{h} \mid \mathrm{n}) \sum_{h^{\prime} \in H}\left\{\left[\delta_{\mathrm{hh}^{\prime}}-\mathrm{f}\left(\mathrm{h}^{\prime} \mid \mathrm{n}\right)\right] \cdot\left[\partial \operatorname{lnl}\left(\mathrm{n} \mid \mathrm{h}^{\prime}\right) / \partial \mathbf{M}_{\mathrm{h}^{\prime}}\right]\right. \\
& \partial \mathbf{M}_{\mathrm{h}^{\prime}}^{\prime} / \partial \mathbf{S}_{\mathrm{h}^{\prime}} \cdot \mathrm{d} \mathbf{S}_{\mathrm{h}^{\prime}} / \mathrm{dt}, \\
& \mathrm{d} \mathbf{S}_{\mathrm{h}} / \mathrm{dt}=\left\{\int_{\mathrm{N}} \mathrm{f}(\mathrm{h} \mid \mathrm{n})\left[\partial \ln \mathrm{l}(\mathrm{n} \mid \mathrm{h}) / \partial \mathbf{M}_{\mathrm{h}}\right] \partial \mathbf{M}_{\mathrm{h}}^{\prime} / \partial \mathbf{S}_{\mathrm{h}},\right.
\end{aligned}
$$

here $\delta_{h h^{\prime}}$ is 1 if $\mathrm{h}=\mathrm{h}^{\prime}, 0$ otherwise. Parameter $\mathrm{t}$ is the time of the internal dynamics of the MF system (like a number of internal iterations). The following theorem was proven.

Theorem. Equations (2) through (4) define a convergent dynamic system MF with stationary states defined by $\max _{\left\{\mathbf{S}_{\mathrm{h}}\right\}} \mathrm{L}$.

In plain language this means that the above equations indeed result in concept-models in the "mind" of the MFT system, which are most similar [in terms of similarity (1)] to the sensory data. Despite a combinatorially large number of items in (1), a computational complexity of the MF method is relatively low, it is linear in $\mathrm{N}$, it could be implemented by a physical system (like the brain) and therefore it may correspond to the working of the mind. These equations describe a 'semantic closure' sustaining its operations; when input signals continuously coming in real time the above equations should be supplemented by a mechanism retaining input signals in working memory for a finite period of time.

\section{Dynamic logic and combinatorial complexity}

Combinatorial complexity of the past algorithms, as indicated in the previous section, is related to a basic structure of a similarity measure (1). It has also been related to the type of logic, underlying various algorithms and neural networks $\left[{ }^{18}\right]$. Formal logic is based on the "law of excluded third", according to which every statement is either true or false and nothing in between. Therefore, algorithms based on formal logic have to evaluate every little variation in data or internal representations as a separate logical statement; a large number of combinations of these variations causes combinatorial complexity. In fact, combinatorial complexity of algorithms based on logic has been related to the Gödel theory: it is a manifestation of the incompleteness of logic in finite systems $\left[{ }^{19}\right]$. Multivalued logic and fuzzy logic were proposed to overcome limitations related to the law of excluded third $\left[{ }^{20}\right]$. Yet the mathematics of multivalued logic is no different in principle from formal logic. Fuzzy logic encountered a difficulty related to the degree of fuzziness: if too much fuzziness is specified, the solution does not achieve a needed accuracy, if too little, it might become similar to formal logic, and adapting the required degree of fuzziness by 'brute force' at every processing step for every combination of models and data would lead to combinatorial complexity. To summarize the above discussion, formal logic starts with exact knowledge (or assumptions) and results in exact deductions, but if exact knowledge is unavailable, trying all kind of assumptions leads to combinatorial complexity. Fuzzy logic starts with fuzzy knowledge (or assumptions) and leads to fuzzy deductions, which may not satisfy the required accuracy. Dynamic logic starts with fuzzy knowledge-assumptions and proceed to exact deductions - much like human mind does.

\section{MFT Hierarchical Organization}

The previous sub-section described a single processing layer in a hierarchical MFT system. An input to each layer is a set of signals $\mathbf{X}(\mathrm{n})$, or in neural terminology, an input field of neuronal activations. An output are the activated models $\mathbf{M}_{\mathrm{h}}\left(\mathbf{S}_{\mathrm{h}}, \mathrm{n}\right)$; it is a set of models or concepts recognized in the input signals. Equations (2-4) describe a loop-process: at each iteration (or internal-time t) the 1.h.s. of the equations contain association variables $\mathrm{f}(\mathrm{h} / \mathrm{n})$ and other model parameters computed at the previous iteration. In other words, the output models "act" upon the input to produce a "refined" output models (at the next iteration). This process is directed at increasing the similarity between the models and signals. It can be described as an internal behavior generated by the models.

The model outputs initiate other actions as well. First, activated models (neuronal axons) serve as input signals to the next processing layer, where more general conceptmodels are recognized or created. Second, concept-models along with the corresponding instinctual signals and emotions may activate behavioral models and generate 
behavior directed into the outside world (a process not contained within the above equations). In general, a higher level in a hierarchical system provides a feedback input into a lower level. For example, sensitivity of retinal ganglion cells depends on the objects and situations recognized higher up in the visual cortex; or a gaze is directed based on which objects are recognized in the field of view. Concept-objects identified at the output of the lower level of MFT system become input signals to the next MFT level which identifies more general concepts of relationships among objects and situations; at the same time more general concepts of understanding identified at a higher level activate behavioral concept-models that affect processes at a lower level. The agent processes, or the loop-processes of model-concept adaptation, understanding and behavior generation continue up and down the hierarchy of the MFT levels.

The MFT operations involves adaptation of multiple models or concepts; these model-adaptation processes are 'agents', to some extent they are independent, yet some model-concept-agents interact when they are associated with the same data pieces. MFT therefore is an intelligent system composed of multiple adaptive intelligent agents, which possess a degree of autonomy yet interact among themselves. Each concept-model-agent along with the similarity measure and behavioral response is a continuous loop of operations, interacting with other agents from time to time; an agent is "dormant" until activated by a high similarity value. When activated, it is adapted to the signals and other agents, so that the similarity increases. Every piece of signal may activate several concepts, or agents, in this way input signals provide evidence for the presence of various objects (or concepts). Agents compete with each other for evidence (data), while adapting to the new signals.

\section{MFT THEORY OF THE MIND}

\section{MFT Dynamics}

Equations (2-4) describe an elementary process of perception or cognition, in which a large number of modelconcepts compete for incoming signals, model-concepts are modified and new ones are formed, and eventually, more or less definite connections [high or low values of $f(h / n)$, varying between 0 and 1] are established among signal subsets on the one hand, and model-concepts on the other. Perception refers to processes in which the input signals come from sensory organs and model-concepts correspond to objects in the surrounding world. Cognition refers to higher levels in the hierarchy where the input signals are concepts activated at lower levels and model-concepts are more complex and correspond to situations and relationships among lower-level concepts.

A salient mathematical property of this processes ensuring a smooth convergence is a correspondence between uncertainty in models (that is, in the knowledge of model parameters) and uncertainty in associations $\mathrm{f}(\mathrm{h} / \mathrm{n})$. In perception, as long as model parameters do not correspond to actual objects, there is no match between models and signals; many models poorly match many objects, and associations remain fuzzy (nor 1 nor 0 ). Eventually, one model (h') wins a competition for a subset $\left\{n^{\prime}\right\}$ of input signals $\mathbf{X}(\mathrm{n})$, when parameter values match object properties, and $f\left(h^{\prime} \mid n\right)$ values become close to 1 for $n \in\left\{n^{\prime}\right\}$ and 0 for $n \notin\left\{n^{\prime}\right\}$. This means that this subset of data is recognized as a specific object (concept). Upon the convergence, the entire set of input signals $\{n\}$ is divided into subsets, each associated with one model-object, uncertainties become small, and fuzzy a priori concepts become crisp concepts. Cognition is different from perception in that models are more general, more abstract, and input signals are the activation signals from concepts identified (cognized) at a lower hierarchical level; the general mathematical laws of cognition and perception are similar in MFT and constitute a basic principle of the the mind organization. Let us discuss relationships between the MFT theory and concepts of the mind developed in psychology, philosophy, linguistics, aesthetics, neurophysiology, neural networks, artificial intelligence, pattern recognition, and intelligent systems.

\section{Elementary Thought-Process, Conscious and Unconscious}

A thought-process or thinking involves a number of subprocesses and attributes, including internal representations and their manipulation, attention, memory, concept formation, knowledge, generalization, recognition, understanding, meaning, prediction, imagination, intuition, emotion, decisions, reasoning, goals, behavior, conscious and unconscious $\left[{ }^{29}, 2\right]$. A "minimal" subset of these processes involves mechanisms for afferent and efferent signals, Grossberg, S. (1988), in other words, bottom-up and top-down signals coming from outside (external sensor signals) and from inside (internal representation signals). According to Carpenter and Grossberg[ $\left[{ }^{21}\right]$ every recognition and concept formation process involves a "resonance" between these two types of signals. In MFT, at every level in a hierarchy the afferent signals are represented by the input signal field $\mathbf{X}$, and the efferent signals are represented by the modeling field signals $\mathbf{M}_{\mathrm{h}}$; resonances correspond to high similarity measures $1(\mathrm{n} / \mathrm{h})$ for some subsets of $\{n\}$ that are "recognized" as concepts (or objects) h. The mechanism leading to the resonances is given by (2-4), and we call it an elementary thought-process. The elementary thought-process involves elements of conscious and unconscious processes, imagination, memory, internal representations, concepts, instincts, emotions, understanding and behavior as further described later.

A description of working of the mind as given by the MFT dynamics was first provided by Aristotle $\left[{ }^{22}\right]$, describing thinking as a learning process in which an a priori form-aspotentiality (fuzzy model) meets matter (sensory signals) and becomes a form-as-actuality (a concept). Jung suggested that conscious concepts are developed by the mind based on genetically inherited structures of the mind, archetypes, 
which are inaccessible to consciousness $\left[{ }^{23}\right]$ and Grossberg suggested that only signals and models attaining a resonant state (that is signals matching models) reach consciousness $\left[{ }^{29}\right]$.

\section{Understanding}

In the elementary thought process, subsets in the incoming signals are associated with recognized model-objects, creating phenomena (of the MFT-mind) which are understood as objects, in other words signal subsets acquire meaning (e.g., a subset of retinal signals acquires a meaning of a chair). There are several aspects to understanding and meaning. First, object-models are connected by emotional signals $\left[{ }^{12},{ }^{24},{ }^{2}\right]$ to instincts that they might satisfy, and also to behavioral models that can make use of them for instinct satisfaction. Second, an object is understood in the context of a more general situation in the next layer consisting of more general concept-models, which accepts as input-signals the results of object recognition. That is, each recognized object-model (phenomenon) sends (in neural terminology, activates) an output signal; and a set of these signals comprises input signals for the next layer models, which 'cognize' more general concept-models. And this process continues up and up the hierarchy of models and mind toward the most general models a system could come up with, such as models of universe (scientific theories), models of self (psychological concepts), models of the meaning of existence (philosophical concepts), models of a priori transcendent intelligent subjects(theological concepts).

\section{Imagination}

Imagination involves excitation of a neural pattern in a visual cortex in the absence of an actual sensory stimulation (say, with closed eyes) $\left.{ }^{[29}\right]$. Imagination was often considered to be a part of thinking processes; Kant $\left[{ }^{25}\right]$ emphasized the role of imagination in the thought process, he called thinking "a play of cognitive functions of imagination and understanding". Whereas the pattern recognition and artificial intelligence algorithms of the recent past would not know how to relate to this $\left[{ }^{26}\right]$, the Carpenter and Grossberg resonance model $\left[{ }^{21}\right]$ and the MFT dynamics both describe imagination as an inseparable part of thinking: imagined patterns are top-down signals that prime the percepting cortex areas (priming is a neural terminology for making neural cells to be more readily excited). In MFT, the imagined neural patterns are given by models $\mathbf{M}_{\mathrm{h}}$. MFT (in agreement with neural data) just adds details to Kantian description: thinking is a play of higher-hierarchical-level imagination and lower-level understanding. Kant identified this "play" [described by (3-6) or (7-12)] as a source of aesthetic emotion; modeling aesthetic emotion in MFT is described later.

\section{The mind vs. Brain}

Historically, the mind is described in psychological and philosophical terms, whereas the brain is described in terms of neurobiology and medicine. Within scientific exploration the mind and brain are different description levels of the same system. Establishing relationships between these description is of great scientific interest. Today we approach solutions to this challenge $\left[{ }^{27}\right]$, which eluded Newton in his attempt to establish physics of "spiritual substance" $\left[{ }^{28}\right]$. General neural mechanisms of the elementary thought process (which are similar in MFT and ART have been confirmed by neural and psychological experiments, this includes neural mechanisms for bottom-up (sensory) signals, top-down "imagination" model-signals, and the resonant matching between the two $\left.{ }^{[29}\right]$. Adaptive modeling abilities are well studied with adaptive parameters identified with synaptic connections $\left[{ }^{30}\right]$; instinctual learning mechanisms have been studied in psychology and linguistics $\left[{ }^{31}\right]$.

\section{Instincts and Emotions}

Functioning of the mind and brain cannot be understood in isolation from the system's "bodily needs". For example, a biological system (and any autonomous system) needs to replenish its energy resources (eat); this and other fundamental unconditional needs are indicated to the system by instincts, which could be described as internal sensors. Emotional signals, generated by this instinct are perceived by consciousness as "hunger", and they activate behavioral models related to food searching and eating. In this paper we are concerned primarily with the behavior of recognition: instinctual influence on recognition modify the objectperception process (3) - (6) in such a way, that desired objects "get" enhanced recognition. It can be accomplished by modifying priors, $\mathrm{r}(\mathrm{h})$, according to the degree to which an object of type $h$ can satisfy a particular instinct. Details of these mechanisms are not considered here, except for a specific instinct considered below.

\section{Aesthetic Emotions and Instinct for Knowledge}

Recognizing objects in the environment and understanding their meaning is so important for human evolutionary success that there has evolved an instinct for learning and improving concept-models. This instinct (for knowledge and learning) is described in MFT by maximization of similarity between the models and the world, (1). Emotions related to satisfaction-dissatisfaction of this instinct are perceived by us as harmony-disharmony (between our understanding of how things ought to be and how they actually are in the surrounding world). According to Kant (1790) $\left[{ }^{10}\right]$ these are aesthetic emotions (emotions that are not related directly to satisfaction or dissatisfaction of bodily needs).

Beauty. Harmony is an elementary aesthetic emotion related to improvement of object-models. Higher aesthetic emotions are related to the development of more complex "higher" models: we perceive an object or situation as aesthetically pleasing if it satisfies our learning instinct, that is the need for improving the models and increasing similarity (1). The highest forms of aesthetic emotions are related to the most general and most important models. According to Kantian 
analysis $\left[{ }^{25}\right]$, among the highest models are models of the meaning of our existence, purposiveness or intentionality, and beauty is related to improving these models: we perceive an object or a situation as beautiful, when it stimulates improvement of these highest models of meaning. Beautiful is what "reminds" us of our purposiveness. The mathematical description of the emotion of beautiful is given by increase of similarity measure related to the most general models of our purpose.

\section{Intuition}

Intuition includes an intuitive perception (imagination) of object-models and their relationships with objects in the world, as well as higher-level models of relationships among simpler models. Intuition involves fuzzy unconscious concept-models, which are in a state of being learned and being adapted toward crisp and conscious models (a theory); such models may satisfy or dissatisfy the knowledge instinct in varying degrees before they are accessible to consciousness, hence the complex emotional feel of an intuition. The beauty of a physical theory discussed often by physicists is related to satisfying our feeling of purpose in the world, that is, satisfying our need to improve the models of the meaning in our understanding of the universe.

\section{Theory Testing and Future Directions}

The general neural mechanisms of the elementary thought process, which includes neural mechanisms for bottom-up (sensory) signals, top-down "imagination" model-signals, and the resonant matching between the two $\left[{ }^{29}\right]$, have been confirmed by neural and psychological experiments (these mechanisms are similar in MFT and ART $\left[{ }^{21}\right]$. Adaptive modeling abilities are well studied and adaptive parameters have been identified with synaptic connections $\left[{ }^{30}\right]$; instinctual learning mechanisms have been studied in psychology and linguistics $\left[{ }^{32}\right]$. Ongoing and future research will confirm, disprove, or suggest modifications to specific mechanisms of model parameterization and parameter adaptation (4), reduction of fuzziness during learning (2), similarity measure (1) as a foundation of aesthetic instinct for knowledge, relationships between psychological and neural mechanisms of learning on the one hand and, on the other, aesthetic feelings of harmony and emotion of beautiful. Differentiated forms of (1) need to be developed for various forms of the knowledge instinct (child development, language learning, emotional intelligence, etc.) Future experimental research needs to study in details the nature of hierarchical interactions: to what extent the hierarchy is "hardwired" vs. adaptively emerging; what is a hierarchy of learning instinct? A theory and experimental data will have to be developed for differentiated form of knowledge instinct and differentiated aesthetic emotions and their role in learning and intelligence.

\section{REFERENCES}

${ }^{1}$ Plato, IV BC, Parmenides, in: Plato, L. Cooper, Oxford Univ. Press, New York, NY, 1997

2 L.I.Perlovsky, 2001, Neural Networks and Intellect, Oxford Univ. Press, New York, NY.

${ }^{3}$ Aristotle, IV BC, Metaphysics, tr. W.D.Ross, in: Complete Works of Aristotle, Ed.J.Barnes, Princeton, NJ, 1995.

${ }^{4}$ L.I.Perlovsky, 1996, Proc. Conf. Intelligent Systems and Semiotics '96. Gaithersburg, MD, v.1, pp. 43-48.

5 I.Kant, 1781. Critique of Pure Reason, tr. J.M.D. Meiklejohn, Willey Book, New York, NY, 1943.

${ }^{6}$ C.G.Jung, 1934, Archetypes of the Collective Unconscious, in: the Collected Works, v.9,II, Princeton Univ. Press, Princeton, NJ, 1969.

7 Abilities of the mind described here, the details of their psychological, biological, and neural mechanisms and content are subject of active research and even the terminology is not yet settled. The two references [2, 8] given here provide sufficient discussion and further references to these details. This section summarizes the main aspects of these abilities that are in agreement with a large body of published work.

8 S.Grossberg, 1982, Studies of Mind and Brain, D.Reidel Publishing Co., Dordrecht, Holland.

${ }^{9}$ Perlovsky, L.I. (2006). Modeling Field Theory of Higher Cognitive Functions. Chapter in A. Loula, R. Gudwin, J.

Queiroz, eds., Artificial Cognition Systems. Idea Group, Sao Paolo, Brazil. In print.

10 I.Kant, 1790, Critique of Judgment, tr. J.H.Bernard, Macmillan \& Co., London, 1914.

${ }^{11}$ Some of the instincts have complex nature [e.g., J.Piaget, The Psychology of the Child, tr. H.Weaver, Basic Books, 2000]; I will not here discuss the differentiated nature of some of the instincts; for an illustration, I will mention that a mechanism of the instinct for hunger is, essentially, the measurement of the level of sugar in blood.

12 S.Grossberg \& D.S.Levine, 1987, Neural dynamics of attentionally modulated Pavlovian conditioning: blocking, inter-stimulus interval, and secondary reinforcement. Psychobiology, 15(3), pp.195-240.

13 J. Searle, 1983, Intentionality : An Essay in the Philosophy of Mind, Cambridge, Univ. Press, Cambridge, GB.

${ }^{14}$ J. Searle, 1992, The Rediscovery of the Mind MIT Press, Cambridge, MA.

15 G.Adelman, 1987, Encyclopedia of Neuroscience. Birkhaüser, Boston, MA.

${ }^{16}$ In fact there are many levels between the retina, visual cortex, and object-models.

17 Perlovsky, L.I. (1996). Mathematical Concepts of Intellect. Proc. World Congress on Neural Networks, San Diego, CA; Lawrence Erlbaum Associates, NJ, pp.1013-16; Perlovsky, L.I.(1997).Physical Concepts of Intellect. Proc. Russian Academy of Sciences, 354(3), pp. 320-323.

18 Perlovsky, L.I. (1998). Conundrum of Combinatorial Complexity. IEEE Trans. PAMI, 20(6) p.666-70. 
${ }^{19}$ Perlovsky, L.I. (1996). Gödel Theorem and Semiotics. Proceedings of the Conference on Intelligent Systems and Semiotics '96. Gaithersburg, MD, v.2, pp. 14-18.

${ }^{20}$ Jang, J.-S.R., Sun, C.-T., and Mizutani, E. (1996). NeuroFuzzy and Soft Computing: A Computational Approach to Learning and Machine Intelligence. Prentice Hall, Upper Saddle River, NJ.

${ }^{21}$ Carpenter, G.A. \& Grossberg, S. (1987). A massively parallel architecture for a self-organizing neural pattern recognition machine, Computer Vision, Graphics and Image Processing, 37, 54-115.

${ }^{22}$ Aristotle, IV BC, Metaphysics, tr. W.D.Ross, Complete Works of Aristotle, Ed.J.Barnes, Princeton, NJ, 1995.

23 Jung, C.G. (1934). Archetypes of the Collective Unconscious. In the Collected Works, v.9,II, Bollingen Series XX, 1969, Princeton University Press, Princeton, NJ.

24 Perlovsky, L.I. (1998). Cyberaesthetics: aesthetics, symbol, and control. Proc. STIS'98: Joint Conf. Science \& Tech. Intelligent Sys. (ISIC/CIRA/ISAS), Gaithersburg, MD.

${ }^{25}$ Kant, I. (1790). Critique of Judgment, tr. J.H.Bernard, Macmillan \& Co., London, 1914.

${ }^{26}$ Newell, A. 1983, Intellectual issues in the history of artificial intelligence. In The study of information, ed. F. Machlup \& U. Mansfield, J. Wiley, New York, NY; M. Minksy, 1988, Society of Mind, Touchstone Books, New York, NY

${ }^{27}$ Grossberg, S. (2000). Linking mind to brain: the mathematics of biological intelligence. Notices of the American Mathematical Society, 471361-1372.

${ }^{28}$ Westfall, R.S. (1983). Never at Rest: A Biography of Isaac Newton. Cambridge Univ Pr., Cambridge.

${ }^{29}$ S. Grossberg, Neural Networks and Natural Intelligence. MIT Press, Cambridge, MA, 1988, S.Zeki, A Vision of the Brain Blackwell, Oxford, England, 1993; W.J. Freeman, Mass action in the nervous system. Academic Press, New York, NY, 1975.

30 C.Koch and I.Segev, Edts., Methods in Neuronal Modeling: From Ions to Networks MIT Press, Cambridge, MA 1998; D.Hebb, Organization of Behavior J.Wiley \& Sons, New York, NY, 1949.

${ }^{31}$ J.Piaget, The Psychology of the Child Tr. H.Weaver, Basic Books, 2000); N.Chomsky, in Explanation in Linguistics, ed. N.Hornstein and D.Lightfoot Longman, London, 1981. Jackendoff, R. (2002). Foundations of Language: Brain, Meaning, Grammar, Evolution, Oxford Univ Pr.; Deacon, T.W. (1998). The Symbolic Species: The Co-Evolution of Language and the Brain. W.W. Norton \& Company.

${ }^{32}$ J.Piaget, The Psychology of the Child Tr. H.Weaver, Basic Books, 2000); N.Chomsky, in Explanation in Linguistics, ed. N.Hornstein and D.Lightfoot Longman, London, 1981. 